\title{
Modelling turbulent skin-friction control using linearized Navier-Stokes equations
}

\author{
C. A. Duque-Daza ${ }^{1,2}$, M. F. Baig ${ }^{1}$, D. A. Lockerby ${ }^{1} \dagger$, S. I. Chernyshenko ${ }^{3}$ \\ and C. Davies ${ }^{4}$ \\ ${ }^{1}$ School of Engineering, University of Warwick, Coventry CV4 7AL, UK \\ 2 Department of Mechanical and Mechatronics Engineering, Universidad Nacional de Colombia, \\ Bogota 111321, Colombia \\ ${ }^{3}$ Department of Aeronautics, Imperial College, London SW7 2AZ, UK \\ ${ }^{4}$ School of Mathematics, Cardiff University, Cardiff CF24 4AG, UK
}

(Received 22 November 2011; revised 3 April 2012; accepted 16 April 2012; first published online 23 May 2012)

Linearized Navier-Stokes equations are solved to investigate the impact on the growth of near-wall turbulent streaks that arises from streamwise-travelling waves of spanwise wall velocity. The percentage change in streak amplification due to the travelling waves, over a range of wave parameters, is compared to published direct numerical simulation (DNS) predictions of turbulent skin-friction reduction; a clear correlation between the two is observed. Linearized simulations at a much higher Reynolds number, more relevant to aerospace applications, produce results that show no marked differences to those obtained at low Reynolds number. It is also observed that there is a close correlation between DNS data of drag reduction and a very simple characteristic of the 'generalized' Stokes layer generated by the streamwise-travelling waves.

Key words: drag reduction, turbulence control

\section{Introduction}

Recent studies of wall-bounded turbulence have shown that spanwise oscillations of the surface (perpendicular to the flow) and streamwise-travelling waves of spanwise wall velocity can bring about a substantial reduction in skin-friction drag. It has been found that these wall-oscillation control approaches have a strongly disruptive/inhibitive effect on the near-wall streak structures (Jung, Mangiavacchi \& Akhavan 1992; Karnidakis \& Choi 2003; Ricco \& Quadrio 2008). Given that it is commonly accepted that near-wall streak structures play a major role in the turbulent regeneration mechanism (Kline et al. 1967), it might be expected that an explanation for the efficacy of such control methods would be readily forthcoming. However, there is still a lack of clarity in our understanding of the physical mechanism(s) underpinning this type of control strategy, which stands in the way of efficient prediction and engineering application.

Choi, $\mathrm{Xu} \&$ Sung (2002) demonstrated, experimentally, drag reduction of up to $45 \%$ using wall oscillations in the spanwise direction; an optimum period 


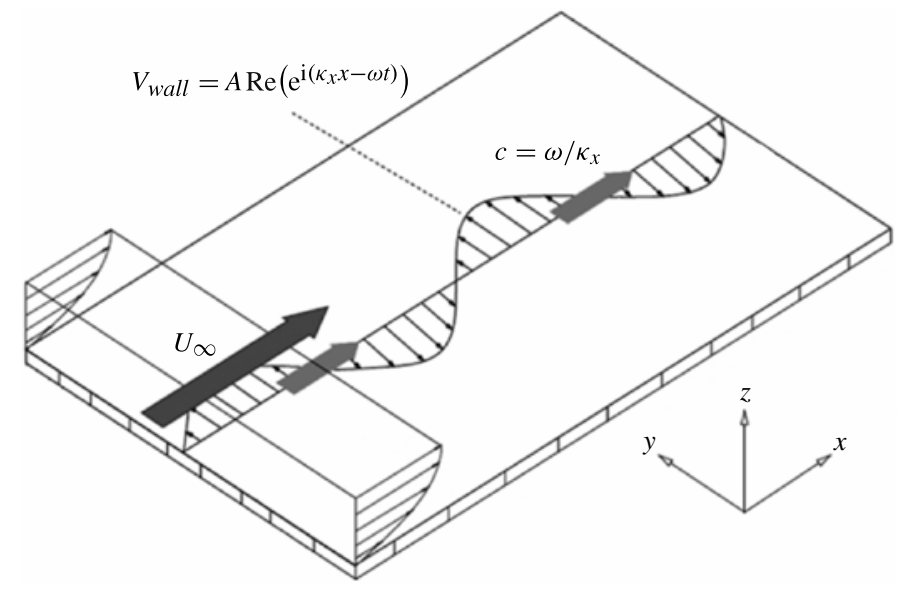

FIGURE 1. Schematic representation of streamwise-travelling waves of spanwise wall velocity. Adapted from Quadrio et al. (2009).

of around $T^{+}=100-125$ was shown to be independent of the wall-oscillation amplitude (here ${ }^{+}$denotes viscous wall units). More recently, Quadrio, Ricco \& Viotti (2009) investigated, numerically, streamwise-travelling waves of spanwise wall velocity: $V_{\text {wall }}=A \sin \left(\kappa_{x} x-\omega t\right)$ where $A$ is the velocity amplitude, $\kappa_{x}$ is a streamwise wavenumber and $\omega$ is the oscillation frequency of the wave. This form of forcing generates waves of spanwise velocity moving (backward or forward) in the streamwise direction with a phase speed $c=\omega / \kappa$ (see figure 1 for a schematic of this actuation). This forcing represents a generalized form of spanwise-wall actuation including the two extreme cases: spanwise oscillation $\left(\kappa_{x}=0\right)$ as investigated by Choi et al. (2002); and stationary streamwise waves of spanwise velocity $(\omega=0)$. Quadrio et al. (2009) found a range of wave parameter combinations for which drag reduction was of the order of $48 \%$ (greater than that achievable using spanwise oscillations or standing streamwise waves of spanwise velocity). They also found that in the $\kappa_{x}-\omega$ parameter space there exists a region where drag increase can occur, corresponding to a constant phase speed $c^{+} \approx 10$. Auteri et al. (2010) performed experiments to confirm the numerical results of Quadrio et al. (2009), and showed that turbulent skin-friction reduction of up to $33 \%$ is achievable for slow velocity forward-travelling waves in a turbulent pipe flow. Moreover, they have also reported that there are combinations of $\kappa_{x}$ and $\omega$ for which an increase in drag can occur; all in consonance with the numerical findings. Despite a number of interesting and thorough studies, it has yet to be satisfactorily explained why skin-friction drag is affected, in the way that it is, by changes in the actuation parameters $\kappa_{x}$ and $\omega$. In this paper we intend to contribute to such an explanation.

The majority of the numerical studies on drag reduction using spanwise wall motion have been performed using direct numerical simulation (DNS). As such, computational limitations have restricted the studies to relatively low Reynolds numbers. In this paper we investigate the behaviour of near-wall streaks using linearized Navier-Stokes equations, allowing us to consider high-Reynolds-number regimes beyond the current capabilities of DNS.

The idea of using linearized Navier-Stokes equations to predict aspects of nonlinear turbulent flows stems from the work of Landahl (1989), who argued that near-wall 
turbulence could be effectively modelled as a linear system driven by spatially and temporally intermittent vorticity bursts originating in the viscous sublayer. Butler \& Farrell (1993) went on to calculate optimal perturbations to a suitably prescribed mean turbulent velocity profile. This work encouraged other approaches to using the linearized Navier-Stokes equations to predict near-wall streaks, e.g. the generalized optimal perturbation (GOP) method of Chernyshenko \& Baig (2005) and the low-order optimization model developed by Lockerby, Carpenter \& Davies (2005). Motivated by the achievements of these earlier studies, we will extend the use of linearized modelling to investigate how near-wall streaks are influenced by streamwise-travelling wave actuation.

Comparisons will be made between our predictions for the linear streak evolution, in the controlled flow, and behaviour that has previously been reported for full DNS studies. We will highlight some particular features for which there is a significant level of agreement. The fact that we have been able to trace an accordance with DNS studies supports the conjecture that our relatively simple linear simulations can be used to shed light on the nature of the physical mechanisms that are responsible for the observed changes in turbulent skin friction. Nevertheless, we are well aware that many researchers have expressed serious concerns about attempts to employ linearized models to capture significant aspects of turbulence. See, for example, the objections that were made by Waleffe (1995) to the assignment of a key role to non-normal linear growth mechanisms in sustaining turbulence. Counter-arguments to these objections were later given by Henningson (1996). In a short paper such as this, it is not feasible or desirable to rehearse all of the controversial issues involved. Instead, we will defer a few further brief remarks until the latter part of the paper, after we have exhibited the interest of the results that were obtained from our linearized simulations.

The paper is organized as follows: $\S 2$ describes the mathematical and numerical formulation used in the study. In particular, $\S 2.1$ briefly outlines the numerical method of solving the linearized Navier-Stokes equations. In $\S 2.2$ we describe the choice of the initial condition independent of the frequency and wavenumber. Section 2.3 explains the choice of the base flow for a given frequency and wavenumber. In $\S 3$ we present results showing how streak amplification is affected by streamwise-travelling waves over a range of actuation parameters, and compare this to DNS data for skinfriction drag reduction; $\S 4$ contains a summary and some concluding remarks.

\section{Mathematical and numerical formulation}

We will solve numerically the initial-value problem for linearized Navier-Stokes equations for different base profiles but with the same initial condition. The base profiles will correspond to a two-parameter family set of travelling-wave wall motion, parameterized with the oscillation frequency and the wavenumber. We will determine the maximum energy amplification of the initial perturbation as a function of the oscillation frequency and the wavenumber and compare it with the turbulent drag reduction calculated from the full Navier-Stokes equations by other authors for the same frequency and wavenumber. In the entire procedure only linearized Navier-Stokes equations will be used.

\subsection{Numerical method}

For the present study, a velocity-vorticity formulation of the Navier-Stokes equations, has been adopted; a brief overview of the formulation is given here, but the interested reader is referred to Davies \& Carpenter (2001) for a detailed exposition. 
The formulation assumes a known base flow solution, represented by the velocity field $\boldsymbol{U}_{b}$, with the corresponding vorticity field $\boldsymbol{\Omega}_{b}$. The total velocity and vorticity fields can then be decomposed as $\boldsymbol{U}_{b}+\boldsymbol{u}$ and $\boldsymbol{\Omega}_{b}+\boldsymbol{\omega}$ where $\boldsymbol{u}=(u, v, w)$ and $\boldsymbol{\omega}=\left(\omega_{x}, \omega_{y}, \omega_{z}\right)$ represent perturbations from the prescribed base flow. Here $u$, $v$ and $w$ are the velocity components in the streamwise $(x)$, spanwise $(y)$ and wall-normal (z) directions. For the known base flow we adopt a combination of a turbulent mean velocity profile in the streamwise direction $(U(z))$ and a generalized Stokes layer, discussed below, in the spanwise direction representing the control, $V(x, z, t)$.

The components of the perturbation flow fields $\boldsymbol{u}$ and $\boldsymbol{\omega}$ may be divided into two distinct sets. The components $\left\{\omega_{x}, \omega_{y}, w\right\}$ are referred to as primary variables, while the remaining components $\left\{u, v, \omega_{z}\right\}$ are secondary variables. The evolution of the three primary variables $\left\{\omega_{x}, \omega_{y}, w\right\}$ is calculated using the following three equations:

$$
\begin{gathered}
\frac{\partial \omega_{x}}{\partial t}+\frac{\partial N_{z}}{\partial y}-\frac{\partial N_{y}}{\partial z}=\frac{1}{R} \nabla^{2} \omega_{x}, \\
\frac{\partial \omega_{y}}{\partial t}+\frac{\partial N_{x}}{\partial z}-\frac{\partial N_{z}}{\partial x}=\frac{1}{R} \nabla^{2} \omega_{y}, \\
\nabla^{2} w=\frac{\partial \omega_{x}}{\partial y}-\frac{\partial \omega_{y}}{\partial x},
\end{gathered}
$$

where $R$ is the Reynolds number, and the convective term is $\boldsymbol{N}=\boldsymbol{\Omega}_{b} \times \boldsymbol{u}+\boldsymbol{\omega} \times \boldsymbol{U}_{b}+\boldsymbol{\omega} \times \boldsymbol{u}$. The system of equations is linearized by omitting $\boldsymbol{\omega} \times \boldsymbol{u}$ from the convective term, above. By deploying integral relationships (derived from the definition of the vorticity and its solenoidal property, together with the vanishing of the perturbations at large distances away from the wall) it is possible to explicitly determine the secondary variables from the primary variables, so that only the above three equations need be considered for the evolution of the perturbations. The fluid is bound by an impermeable wall at $z=0$, where the no-slip condition is applied by enforcing integral constraints on the vorticity.

The streamwise and wall-normal directions are discretized using a second-order finite-difference and mapped-domain Chebyshev polynomial expansion, respectively. In the spanwise direction we perform a Fourier decomposition, and as the equations are linear, the individual modes can be solved independently (i.e. as separate twodimensional calculations). Perturbations thus have the form:

$$
\boldsymbol{u}=(\bar{u}, \bar{v}, \bar{w}) \mathrm{e}^{\mathrm{i} \beta y}, \quad \boldsymbol{\omega}=\left(\bar{\omega}_{x}, \bar{\omega}_{y}, \bar{\omega}_{z}\right) \mathrm{e}^{\mathrm{i} \beta y},
$$

where $\beta$ is the spanwise wavenumber. Here, where simulations were conducted for streamwise-travelling waves of spanwise wall motion, it was convenient to apply periodic boundary conditions at the upstream and downstream ends of the computational domain, with a total domain length set equal to the streamwise wavelength of the control wave. Ninety two Chebyshev polynomials and 100 grid points per wavelength were found to give adequate spatial resolution (in terms of generating grid-independent results) for the simulations presented in this paper. It may be noted that the imposition of a streamwise periodicity that matches that of the control wave rules out the possibility of simulating the development of spatially detuned modes of disturbance, which might appear in a more generalized Floquet theory. However, this is not a major drawback, since we are predominantly interested in modelling the transient growth of streaks, rather than Floquet modes that would grow or decay at a constant rate. 


\subsection{Selection of the initial condition}

Transient growth (characterized by inviscid algebraic growth followed by viscous decay) is a mechanism whereby small perturbations can interact with a base shear profile to create much more energetic disturbances; these disturbances can take the form of longitudinal vortices at the start of the motion creating streamwise streaks by the lift-up mechanism and eventually decaying with time (see, for example, Butler \& Farrell 1992, Henningson, Lundbladh \& Johansson 1993 and Cossu, Pujals \& Depardon 2009). In a developed turbulent flow such initial conditions, representing vortex structures with a large degree of spatial coherence, need not occur, of course. The nature of the relationship between transient growth solutions and developed turbulent flows is discussed in detail in Chernyshenko \& Baig (2005).

In order to obtain an appropriate initial condition for the present study we follow a method similar to the low-order optimization method of Lockerby et al. (2005) but with a solution norm proposed by Chernyshenko \& Baig (2005). Here, we specify an initial condition on the perturbation field that produces a near-optimal response to an uncontrolled steady turbulent mean profile. The mean profile that is used, $U(z)$, combines a Musker profile from the wall to the overlap region and a Coles wake function for the outer part; see Nagib \& Chauhan (2008) for further details.

What separates the approach that we take here from a true optimal perturbation method is that the optimization is performed over a parameterized set of initial conditions, making it more numerically efficient, at the expense of being, strictly speaking, suboptimal. This is one of the reasons why we label our modelling as being low order. The initial condition that we chose is of the form:

$$
u(0)=0, \quad w(0)=z^{2} \mathrm{e}^{-b z^{2}} \mathrm{e}^{\mathrm{i} \beta y}
$$

where the $v$-component is determined directly from continuity, and the parameters $b$ and $\beta$ are found via optimization. The form of the initial condition has been selected so as to be comparatively simple (only two parameters) while satisfying the perturbation boundary conditions at $z=0$ and $z \rightarrow \infty$. It is also similar in its basic form to the expected optimal perturbation, namely an elongated streamwise vortex. It may be noted that, for any given value of the parameter $b$, the wallnormal velocity component of the initial condition obtains its maximum amplitude at a distance $z_{b}=1 / \sqrt{b}$ away from the wall.

If we perform an optimization based on a target of maximum energy amplification within a restricted time frame $\left(t^{+}<80\right.$, as per Butler \& Farrell 1993) we obtain an optimum spanwise wavelength $\lambda^{+}=2 \pi / \beta^{+}=104$, having a peak $u$-component velocity occurring at $z^{+}=18.8$. This is very close to that reported by Butler $\&$ Farrell (1993) using the optimal perturbation approach: $\lambda^{+}=110$ with a peak at $z^{+} \approx 19$. This confirms that our low-order-optimization method, while being faster, provides a good approximation to results that can be obtained from an exact optimization.

For the remainder of this paper, we will use a different 'measure' for gauging the intensity of the streaks that develop in the uncontrolled flow. This new measure was first employed within the GOP method developed by Chernyshenko \& Baig (2005). Optimizations based upon it have the advantage that they are known to provide physically valid results without any requirement to specify an optimization time frame (though, when it is more convenient in computations to do so, a choice of time frame can still be made). The perturbation energy associated with the initial condition is integrated over the entire volume of the flow domain and kept fixed, but the amplification is measured by computing the perturbation energy averaged over a 
particular wall-parallel plane, given by $z=z_{p}$. We thus use a planar form of measure for the perturbation output, as opposed to a volume-based measure, which is used for the input. This allows us to specify a wall-normal distance where the streak characteristics need to be considered. Here, we select a near-wall (wall-parallel) plane at $z_{p}^{+}=12$; the motivation for making this particular choice of position is indicated later.

The parameters that give the optimal configuration when the initial condition is optimized in the manner that has just been described are: $\lambda^{+}=2 \pi / \beta^{+}=74.4$ and $b^{+}=0.00325$, which corresponds to $z_{b}^{+}=17.5$. The value for $\lambda^{+}$is very close to the result that was obtained by Chernyshenko \& Baig (2005) using a planar output measure at the same distance from the wall. (This was for the case, as it is here, where the energy of the initial condition was not weighted by the normal Reynolds stress.) Note that, for the simulations that we shall report which involve streamwise-travelling waves, we do not perform any re-optimization, i.e. the initial condition is kept the same throughout our study of the controlled flow. The reason for doing this is that we wish to find as simple a method as possible for assessing the impact of the spanwise wall motion; we thus focus on the effect on the transient development for forms of initial disturbance that would trigger streaks with the largest measure in the uncontrolled flow.

\subsection{Selection of the base flow}

It has been shown by Quadrio et al. (2009) in DNS of plane channel turbulent flow, and also experimentally by Auteri et al. (2010), that streamwise-travelling waves of spanwise wall velocity (as depicted in figure 1) generate a thin, unsteady, streamwisemodulated Stokes layer which can bring about both drag reduction and drag increase depending upon the actuation parameters $\kappa_{x}$ and $\omega$ (as defined earlier). This spanwise oscillatory flow has been described as being a generalized Stokes layer by Quadrio \& Ricco (2011).

The thrust of the current study is to observe, by as simple means as possible, the effects of the spanwise wall motion on linear streak growth. In keeping with the aim of simplicity, we consider a base profile that consists of a prescribed uncontrolled turbulent profile $U(z)$ (in the streamwise direction) together with an unsteady generalized Stokes layer $V(x, z, t)$ in the spanwise direction. It should be noted that the use of such superposition would be strictly correct if the turbulent profile $U(z)$ arose from an exact parallel flow solution of the Navier-Stokes case. However, since this cannot in fact be the case, an approximation is implicitly being made when the generalized Stokes layer is simply added along the spanwise direction, orthogonal to the turbulent mean flow. (Some further brief comments about the validity of using such a base flow model are included immediately below.)

As a preliminary step we need to derive a numerical solution for the generalized Stokes layer with an imposed spanwise velocity at the wall of the form: $V_{\text {wall }}=$ $A \operatorname{Re}\left(\mathrm{e}^{\mathrm{i}\left(\kappa_{x} x-\omega t\right)}\right)$. As was noted in Quadrio \& Ricco (2011), the governing Navier-Stokes equations are simplified substantially if we assume that terms involving $y$-derivatives are null (as there is no variation in the spanwise direction), and the wall-normal pressure gradient is negligible. The $y$-momentum equation can then be solved independently, to directly determine the spanwise velocity component $V$, given any prescribed turbulent profile $U$. It reduces to the form:

$$
\frac{\partial V}{\partial t}+U \frac{\partial V}{\partial x}=\frac{1}{R}\left(\frac{\partial^{2} V}{\partial x^{2}}+\frac{\partial^{2} V}{\partial z^{2}}\right),
$$


where $R$ is the appropriate Reynolds number. The convective term is responsible for a one-way coupling between $U$ and $V$. Introducing the variable $\zeta=x-c t$ with $c=\omega / \kappa_{x}$, and then assuming that the spanwise velocity takes the form $V=A \operatorname{Re}\left(\mathrm{e}^{\mathrm{i} \kappa_{x} \zeta} \bar{V}(z)\right)$, where $\bar{V}(z)$ is a complex-valued profile function, the above equation yields

$$
\left((U-c) \mathrm{i} \kappa_{x} R+\kappa_{x}^{2}\right) \bar{V}(z)=\bar{V}^{\prime \prime}(z) .
$$

This ordinary differential equation, with the conditions $\bar{V}(0)=1$ and $\lim _{z \rightarrow \infty} \bar{V}(z)=0$, can be cast as a boundary-value problem and solved numerically using a finitedifference discretization. In conjunction with the form for $V$ defined above, this allows us to calculate the spanwise velocity component of the base flow, $V(x, z, t)$, at each spatial grid point and as a function of time, for any given combination of $\omega$ and $\kappa_{x}$. The Stokes solution thus obtained uses a laminar flow assumption, but it can be argued that this is acceptable for turbulent flows because the dominant viscous terms are expected to be much larger in magnitude compared to the Reynolds stress terms, as explained in Ricco \& Quadrio (2008). (Note that in the subsequent discussion, we will drop the overbar when referring to the complex-valued profile function $\bar{V}(z)$.)

\section{Results and discussion}

We performed numerical simulations of linear streak growth in a turbulent boundary layer flow at $R e_{\tau}=200$ (a Reynolds number based on wall shear stress and channel half-width), with control using streamwise-travelling waves over a range of $\kappa_{x}^{+}$and $\omega^{+}$. A constant wave velocity amplitude $A^{+}=12$ was taken, so as to match the configuration that was studied in the DNS experiments conducted by Quadrio et al. (2009) for a turbulent channel flow. (Note that, though we report results obtained for a turbulent boundary layer, we were also able to model streak development for the channel flow case using our simulation code, by making suitable amendments to the base flow profile and taking the boundary layer edge to correspond to the channel centreline. This gave essentially the same results, as might be expected because neither the streaks nor the oscillatory control flow had any significant protrusion into the outermost part of the boundary layer.)

For every point in the parametric space, the maximum (over time) of the energy amplification of the initial condition (that is the maximum of the ratio of the energy of the perturbation in the entire flow to its initial value) is calculated. This is compared to the energy amplification of the initial condition without the streamwise-travelling waves, and a percentage change, $\epsilon$, is calculated. A parameter-space map of $\epsilon$ is plotted in figure 2(b); for comparison, in figure 2(a), we have reproduced a map of skin-friction drag change from the DNS of Quadrio et al. (2009). The red regions in the map for the skin-friction indicate a drag reduction (a maximum decrease being $\approx 48 \%$, which occurs near $\omega^{+}=0.018, \kappa_{x}^{+}=0.0075$ ); the blue regions indicate a drag increase (a maximum increase being $\approx 20 \%$ ). There are a number of striking similarities between the two plots. The red regions in figure $2(b)$, indicating maximum streak amplification reduction, generally correlate with regions of maximum drag reduction (the red regions in figure $2 a$ ). This similarity is both in terms of approximate position as well as relative magnitude between different regions. The maximum percentage change in streak growth amplification in figure $2(b)$ is approximately $50 \%$, which is again comparable to that found in DNS for drag reduction. However, it should be pointed out that an optimum parameter combination for the maximum suppression of streak growth is not found within the ranges plotted in figure $2(b)$, 
whereas an optimum drag reduction can be identified for the DNS results shown in figure $2(a)$.

There is a diagonal corridor in the actuation parameter space where instabilities (or very large increases in the disturbance amplification, at least) were discovered in the linearized Navier-Stokes simulations; this is illustrated in figure 3. The regions of high growth in the disturbance energy were found to correlate closely with the (blue) region of drag increase seen in figure 2(a). We observed that instabilities developed more readily from initial conditions that were localized in $x$, but even with the initial conditions that are considered here (which are taken to be streamwise invariant), instabilities still became manifest after a sufficient period of time. When we constructed the contour plot of figure $2(b)$, it was convenient to stop the simulations after a fixed time period $t^{+}=10$ had elapsed, so as to avoid having to fully incorporate the large (and potentially unbounded) growth for parameter values where there was highly unstable behaviour. The time interval was chosen to be long enough to categorize the suppression of streak growth where instability was absent, but sufficiently short to make it possible to disregard the large amplifications that would otherwise have been found in parameter ranges where instability was known to develop. As a result, all of the contour values that are plotted in figure $2(b)$ represent streak growth suppression (there is no region of streak growth increase), with a minimum change being approximately $10 \%$. Nevertheless, even though the unstable development does not become apparent within the curtailed time frame, it is still possible to see a vestige of the instability in the plotted contours. This occurs along an upward diagonal, where there is relatively weak streak suppression (low $\epsilon$ ); the slope angle of this diagonal is similar to the angles that bound the drag-increase corridor region in figure $2(a)$.

We have repeated the simulations that were presented in figure $2(b)$ using a turbulent boundary layer profile for a much higher Reynolds number $R e_{\tau}=2594$, which corresponds to a displacement-thickness Reynolds number $R e_{\delta^{*}}=10^{4}$. The results are shown in figure $4(a)$. The magnitude of the streak amplification change generally remains within $\sim 5 \%$ of that which was obtained for the lower Reynolds number case, as can be seen by making a direct comparison between figures $2(b)$ and $4(a)$, the contour maps are very much the same. This consistency between the simulation results strongly suggests that the effect of the spanwise flow actuation on the near-wall streaks is independent of the Reynolds number, when a correct scaling is made with inner units. Of course, any such conclusion is subject to the validity of the simplifying approximations that were made in using the linear streak model with a prescribed mean-flow turbulent profile. Notwithstanding this, there are positive implications for the use of the wall-motion flow control scheme in aerospace applications, where the high Reynolds number results are more pertinent. The linearized simulation results indicate that there may be no marked deterioration in the possible skin-friction reduction when the Reynolds number is increased above the relatively low values that have been considered in previous studies. However, this optimistic expectation should be balanced by reservations regarding the potential for there to be an increasing role for large-scale structures in the turbulent boundary layer when the Reynolds number is higher. (See, for example, Marusic et al. 2010.) We have not made any explicit attempt to model or identify such structures, which would be anticipated to develop much further away from the wall than any of the streaks that we have focused upon.

It is important to note that predictions for engineering purposes, made using the linear model that we have described, will always require some form of empirical 

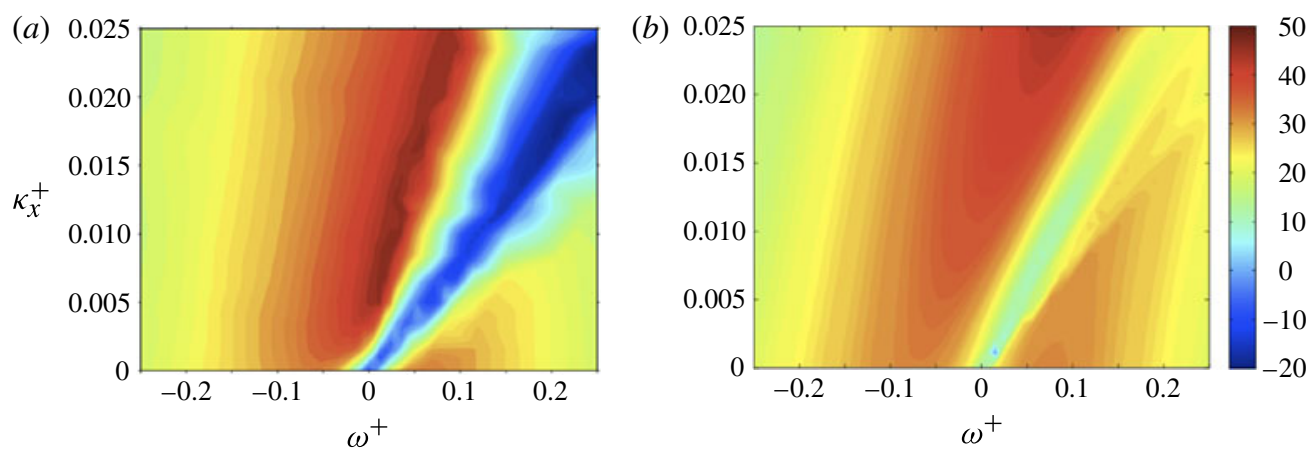

FIGURE 2. Contour plots over the actuation parameter space $\left(\omega^{+}, \kappa_{x}^{+}\right)$of: $(a)$ percentage drag reduction as calculated by DNS, Quadrio et al. (2009); (b) percentage change in streak amplification $(\epsilon)$ as calculated by the linearized Navier-Stokes equations.

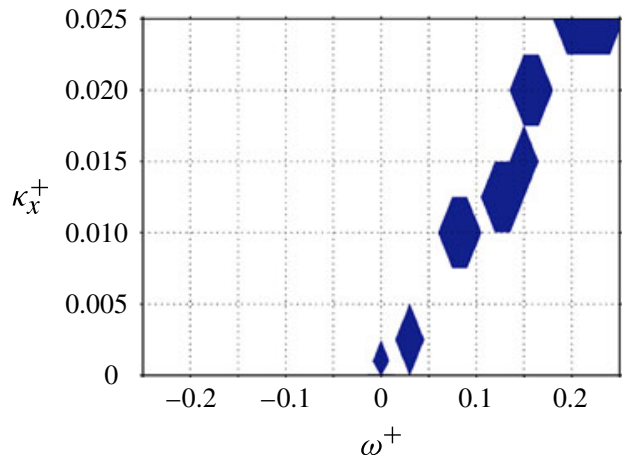

FIGURE 3. Regions of significant streak amplification increase ( $>50 \%)$ occurring over long time scales $\left(t^{+}<300\right)$ due to streamwise-travelling wave actuation. Results generated for a localized three-dimensional initial condition considered at a range of streamwise positions.
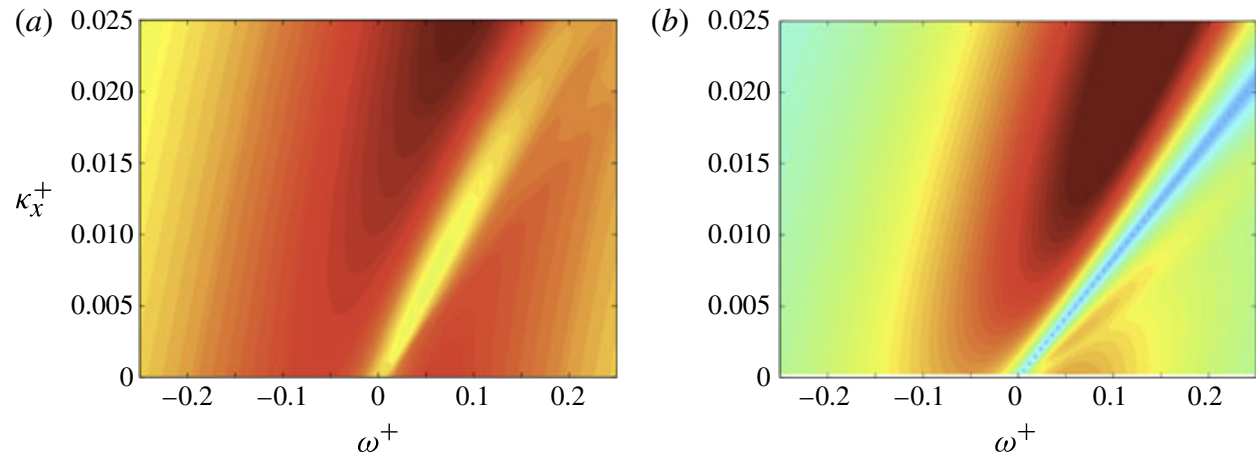

FIgURE 4. (a) Contour plot of amplification factor over actuation parameter space $\left(\omega^{+}, \kappa_{x}^{+}\right)$, as calculated using the linearized Navier-Stokes equations with $\operatorname{Re}_{\tau}=2594$. (b) Corresponding plot of $\left|V^{\prime \prime}\right|$ for $z^{+}=12$. The colouring of the contour levels in $(a)$ is the same as that of the plots in figure 2, but there is a scaling by a factor $O\left(10^{3}\right)$ in $(b)$. 
calibration in order to be reliable. For example, as described earlier, the initial condition that was adopted in this study requires an optimization plane $z=z_{p}$ to be specified for the GOP. Here, we have taken $z_{p}^{+}=12$, motivated by an empirical expectation for the likely location of dynamically significant streaks. If the height of the plane had been chosen very much differently, the contour scales (if not the form) of the maps plotted in figures $2(b)$ and $4(a)$ would have been altered.

The most important conclusion to be drawn from the results presented above is that the main features of the DNS drag-reduction map shown in figure $2(a)$ can be obtained by solving the linearized Navier-Stokes equations in combination with the prescribed basic flow that we have considered. Aside from making the inference that the mechanism which is responsible here for the drag reduction is (at least in some part) linear, we refrain from suggesting any further physical interpretation of the result or attempting a physical description of how the supposed underlying linear process is constructed. However, given that the correlation does exist, it follows that information regarding the main features of the DNS drag-reduction map might somehow be contained within the prescribed basic flow state. To see if this is in fact the case, we have investigated some particular properties of the oscillatory spanwise flow that might have a significant influence on the streak development in the linear model. One property of interest is the wall-normal second derivative of the velocity profile $V^{\prime \prime}=\mathrm{d}^{2} V / \mathrm{d}^{2} z$, since a term involving this derivative features directly in the linearized equation for the streamwise vorticity transport. In figure $4(b)$ a contour map of $\left|V^{\prime \prime}\right|$, evaluated at the previously selected wall-normal position $z^{+}=12$, has been plotted as a function of $\kappa_{x}^{+}$and $\omega^{+}$. The contour values were calculated using an approximate analytical expression for $V$ (see Quadrio \& Ricco 2011). It may be seen that the plot displays a striking qualitative resemblance to the drag-reduction map of Quadrio et al. (2009), reproduced in figure 2(a), which suggests that there is some correlation between zones of larger magnitudes of $\left|V^{\prime \prime}\right|$ and turbulent drag reduction. The light-blue corridor in the map represents $\left|V^{\prime \prime}\right| \approx 0$ and perhaps marks out a zone of inflectional instability, corresponding to the corridor of drag increase in figure 2(a). The precise nature of such an instability, and the role of $\left|V^{\prime \prime}\right|$ in streak growth suppression needs to be the subject of a more in-depth study.

More generally, there is an urgent requirement for further theoretical work to provide a stronger underpinning for the linearized modelling approach that we have adopted in the current paper. Our relatively simple approach appears to yield results that provide a viable starting point for the prediction of drag reduction. This is despite the fact that only a single adjustable parameter is contained in the model, namely the height $z_{p}^{+}=12$ of the near-wall (wall-parallel) plane, which is chosen to measure the sizes of the output disturbances. But even this parameter was effectively fixed by first considering the optimization results for the case with no prescribed wall motion.

Some researchers may regard the apparent fit between the linearized simulation results and DNS studies as merely a happy coincidence. At the time of writing, there is no general consensus as to whether important aspects of turbulence in boundary layers can be truly accounted for in terms of linear transient growth mechanisms, for structures that are located principally in an inner region near the wall. Although we would claim some success from having adopted an approach along such lines, we also recognize that alternative models and descriptions are being advanced and elaborated. Typically, these take nonlinear vortex-structure interactions as being key to the sustainment of turbulence. Further references can be found in the recently conducted review of wall-bounded turbulent flows by Marusic et al. (2010), where it is noted that there is a current widespread dichotomy amongst the views taken 
by researchers, concerning the nature of the time-dependent interactions that can be maintained between various possible flow structures.

\section{Summary}

In this paper we have used linearized Navier-Stokes equations to investigate streak growth characteristics in the presence of streamwise-travelling waves of spanwise wall velocity. A correlation appears to exist between skin-friction reduction seen in DNS studies and the percentage change in streak amplification calculated from the linearized Navier-Stokes equations. The strong implication is that a linear mechanism plays a fundamental role in causing drag reduction via streamwise-travelling waves. The streak growth characteristics in the actuated flow were also shown to be effectively independent of the Reynolds number for the mean turbulent profile that was adopted. Finally, we have shown that at least one basic property of the spanwise velocity profile generated by streamwise-travelling waves correlates well with the DNS prediction of skin-friction drag reduction.

\section{Acknowledgements}

The authors would like to acknowledge the financial support from EPSRC through grant EP/G060215/1, together with Airbus Operations Ltd and EADS UK Ltd. The authors would also like to thank Professor M. Quadrio for helpful discussions and for providing the DNS data used to generate figure $2(a)$ in this paper.

\section{REFERENCES}

Auteri, F., Baron, A., Belan, M., Campanardi, G. \& Quadrio, M. 2010 Experimental assessment of drag-reduction by travelling waves in a turbulent pipe flow. Phys. Fluids 22, 115103.

Butler, K. M. \& FARrell, B. F. 1992 Three-dimensional optimal perturbations in a viscous shear flow. Phys. Fluids 4, 1637-1650.

Butler, K. M. \& FARrell, B. F. 1993 Optimal perturbations and streak spacing in wall-bounded turbulent shear flows. Phys. Fluids 4, 774-777.

Chernyshenko, S. I. \& BAig, M. F. 2005 The mechanism of streak formation in near-wall turbulence. J. Fluid Mech. 544, 99-131.

Choi, J. I., XU, C. X. \& Sung, H. J. 2002 Drag-reduction by spanwise wall-oscillation in wall-bounded flows. AIAA J. 40, 842-850.

Cossu, C., Pujals, G. \& Depardon, S. 2009 Optimal transient growth and very large-scale structures in turbulent boundary layers. J. Fluid Mech. 619, 79-94.

Davies, C. \& CARpenter, P. W. 2001 A novel velocity-vorticity formulation of the Navier-Stokes equations with application to boundary layer disturbance evolution. J. Comput. Phys. 172, $119-165$.

Henningson, D. S. 1996 Comment on 'transition in shear flows. Nonlinear normality versus non-normal linearity'. Phys. Fluids 8, 2257-2258.

Henningson, D. S., Lundbladh, A. \& Johansson, A. V 1993 A mechanism for bypass-transition from localized disturbances in wall-bounded shear flows. J. Fluid Mech. 250, 169-207.

Jung, W. J., Mangiavacchi, N. \& Akhavan, R. 1992 Suppression of turbulence in wall-bounded flows by high-frequency spanwise oscillations. Phys. Fluids A 4, 1605-1607.

KARNidAKIS, G. E. \& CHOI, K.-S. 2003 Mechanisms on transverse motions in turbulent wall-flows. Annu. Rev. Fluid Mech. 35, 45-62.

Kline, S. J., Reynolds, W. C., Schraun, F. A. \& Runstadler, P. W. 1967 The structure of turbulent boundary layers. J. Fluid Mech. 30, 741-773. 
LANDAhL, M. T. 1989 Boundary layer turbulence regarded as a driven linear system. Physica D 37, $11-19$.

Lockerby, D. A., Carpenter, P. W. \& Davies, C. 2005 Control of sublayer streaks using microjet actuators. AIAA J. 43, 1878-1886.

Marusic, I., McKeon, B. J., Monkewitz, P. A., Nagib, H. M., Smits, A. J. \& SREENIVASAN, K. R. 2010 Wall-bounded turbulent flows at high Reynolds numbers: recent advances and key issues. Phys. Fluids 22, 065103.

NAGib, H. M. \& Chauhan, K. A. 2008 Variations of Von-Kármán coefficient in canonical flows. Phys. Fluids 20, 101518.

QUADRIO, M. \& RicCO, P. 2011 The laminar generalized Stokes layer and turbulent drag-reduction. J. Fluid Mech. 667, 135-157.

QuAdrio, M., Ricco, P \& ViotTi, C. 2009 Streamwise travelling waves of spanwise wall-velocity for turbulent drag-reduction. J. Fluid Mech. 627, 161-178.

Ricco, P. \& QUADRIO, M. 2008 Wall-oscillation conditions for drag-reduction in turbulent channel flow. Intl J. Heat Fluid Flow 29, 601-612.

WALEFFe, F. 1995 Transition in shear flows. Nonlinear normality versus non-normal linearity. Phys. Fluids 7, 3060. 